\title{
Waste to Fungi
}

\author{
An EPS@ISEP 2019 Project \\ Alexander Winter ${ }^{1}$, Erendiro Pedro ${ }^{1}$, Julia Ślasko ${ }^{1}$, Julien Battaglini ${ }^{1}$, Mäike Faelker ${ }^{1}$. Ronald Kivipelto ${ }^{1}$, \\ Abel J. Duarte ${ }^{1,4}$, Benedita Malheiro ${ }^{1,3}$, Cristina Ribeiro ${ }^{1,2}$, Jorge Justo ${ }^{1}$, Manuel F. Silva ${ }^{1,3}$, Paulo \\ Ferreira ${ }^{1}$ and Pedro Guedes ${ }^{1}$ \\ ${ }^{1}$ ISEP / PPorto - School of Engineering, Porto Polytechnic, Porto, Portugal \\ ${ }^{2}$ INEB, Porto, Portugal \\ ${ }^{3}$ INESC TEC, Porto, Portugal \\ ${ }^{4}$ REQUIMTE / LAQV, ISEP, Porto, Portugal \\ epsatisep@gmail.com
}

\begin{abstract}
This paper describes the journey of a multinational and multidisciplinary team enrolled in the European Project Semester (EPS) at the Instituto Superior de Engenharia do Porto (ISEP) during the spring semester of 2019. The team embraced the idea of repurposing coffee leftovers to cultivate oyster mushrooms and benefited from the background diversity of the team members as well as from newly acquired marketing, sustainability and design ethics skills to consolidate and strengthen the overall feasibility of the project. The project was set to design, develop and test grey oyster mushroom growth kits with an automated monitoring system, using coffee grounds as growing substrate and complying with the applicable regulations and pre-defined requirements. The ulterior aims of the project were to reconnect people with the food they eat and to disseminate sustainable food production processes, which are not only healthy but environmentally friendly. To achieve these goals, the team developed a circular economy business model where grey oyster mushroom growth kits reuse coffee grounds as growing beds and food buckets as containers. The designed growth kits include a controlled fruiting chamber with an integrated monitoring system. This allows easy domestic cultivation, monitoring through a smart phone. Moreover, the proposed solution contemplates information sharing on the mushroom cultivation process, monitoring system and recipes as well as the maintenance of a dedicated discussion forum. Tests have been conducted to test the concept, cultivation process, monitoring system and fruiting chamber from the incubation of

\footnotetext{
Permission to make digital or hard copies of all or part of this work for personal or classroom use is granted without fee provided that copies are not made or distributed for profit or commercial advantage and that copies bear this notice and the full citation on the first page. Copyrights for components of this work owned by others than ACM must be honored. Abstracting with credit is permitted. To copy otherwise, or republish, to post on servers or to redistribute to lists, requires prior specific permission and/or a fee. Request permissions from Permissions@acm.org.

TEEM'19, October 16-18, 2019, León, Spain

(C) 2019 Association for Computing Machinery.

ACM ISBN 978-1-4503-7191-9/19/10..\$15.00

https://doi.org/10.1145/3362789.3362921
}

mycelium all the way to the harvesting. Results show the feasibility of creating a business based on the devised concept.

\section{CCS CONCEPTS}

- Social and professional topics $\rightarrow$ Model curricula; - Social and professional topics $\rightarrow$ Sustainability; • Social and professional topics $\rightarrow$ Project and people management; - Social and professional topics $\rightarrow$ Codes of Ethics;

\section{KEYWORDS}

Circular Economy, Education for Sustainability, Engineering Capstone Project, European Project Semester, Project-based Learning.

\section{ACM Reference format:}

Alexander Winter, Erendiro Pedro, Julia Ślasko, Julien Battaglini, Mäike Faelker. Ronald Kivipelto, Abel J. Duarte, Benedita Malheiro, Cristina Ribeiro, Jorge Justo, Manuel F. Silva, Paulo Ferreira and Pedro Guedes. 2019. Waste to Fungi: An EPS@ISEP 2019 Project. In Proceedings of the Seventh International Conference on Technological Ecosystems for Enhancing Multiculturality (TEEM 2019) (León, Spain, October 16-18, 2019), ACM, New York, NY, USA, 8 pages. https://doi.org/10.1145/3362789.3362921

\section{Introduction}

The European Project Semester (EPS) is a one semester capstone project or internship program offered to engineering, product design and business under-graduates by 19 European engineering schools (http://europeanprojectsemester.eu), including the Instituto Superior de Engenharia do Porto (ISEP). EPS@ISEP challenges students from multiple educational backgrounds and nationalities to join their competencies to solve multidisciplinary real-life problems. The programme implements project based learning, multicultural teamwork and multidisciplinary problem solving to prepare undergraduates to their future profession. The syllabus comprises one central project module and five support modules, covering team building and project management, energy 
and sustainable development, ethics and deontology, marketing and communication and local language and culture [1]. Team building occurs in the first week to promote the establishment of intra-team connections. Apart from project management and local language and culture, the remaining support modules are short intensive seminars or workshops (up to $24 \mathrm{~h}$ ) intended to make the teams consider the different dimensions involved in the design and development of a solution, namely, economics, ethics, marketing, sustainability and technology, and to support the creation of the mandatory deliverables, including the project wiki, report, presentation, poster, paper, leaflet and video and the prototype manual. The project module revolves around the weekly meetings between the panel of multidisciplinary advisers and each team. Project briefs are open-ended and regard multidisciplinary problems, imposing the compliance with international standards, applicable regulations and the specified budget and recommending the reuse of existing materials and components and the adoption of open source solutions. The panel coaches the teams through brainstorming, promoting sustainable and ethically aligned design while developing sound and innovative technologic solutions [2].

In the spring of 2019, an international team composed of six students, a product designer student from Switzerland, an Angolan electrical and computer engineering student, a business and technology student from Poland, an electrical engineering student from Estonia, a general engineering student from France, and a media technology student from Germany, decided to develop "Waste to Fungi", a sustainable mushroom farm using coffee leftovers. Coffee is a massive industry, worth over 50 billion USD in 2019 [3]. However, when coffee is made, since less than $1 \%$ of the biomass ends up in the cup, $99 \%$ of the biomass is wasted [4]. Coffee leftovers also known as coffee grounds are still full of nutrients and constitute a very good growing medium for oyster mushrooms. The overall objective of this project was: $(i)$ to explore the possibility to create a sustainable business based on coffee grounds collection and their reuse as a growing medium for oyster mushrooms; and (ii) to develop an optimised proof-of-concept growing chamber.

This paper presents the entire process the team went through in the spring semester of 2019 and is divided into seven sections: the second and third sections detail the problem, marketing, sustainability and ethics involved, the fourth section focuses on the design and development of the solution, the sixth presents the tests and results and, finally, the last section draws the main conclusions of the project.

\section{Problem}

According to Statista, 40 tons of coffee are consumed annually in Portugal [5], leaving behind 39.6 tons of wasted biomass. Moreover, the sad reality is coffee beans travelled thousands of miles to end up as waste, thrown away by coffee shops.

Mushrooms are notoriously hard to grow because of inconsistent results. This is partly due to the mass production of low-quality growth kits, which tend to give unreliable results. Even in the wild, mushroom growth is very unpredictable. However, by using the correct knowledge and relatively simple techniques, it is possible to cultivate mushrooms on both an industrial and home scale.

Similar to the fruits produced by a tree, mushrooms are the reproductive fruits of a dense root-like network of cells known as mycelium. Mycelium, when faced with environmental stress, switches into survival mode. In this mode, it produces mushrooms to release spores into the wind and, ultimately, to find a better place to live [6]. Fortunately, man is able to recreate and manage the life cycle of mushrooms to harvest good crops of edible mushrooms. Mushroom farming can be expensive since it relies on very strict growing conditions (high moisture, high oxygen and the right temperature) to cause mushrooms to grow.

Pleurotus Ostreatus, also known as grey oyster mushrooms, is a very resilient and tasteful mushroom variety which can grow in many types of agricultural waste [4]. The most common materials used to grow oyster mushrooms are freshly cut hardwood logs or straw. Growing on logs can be difficult and take several years before a first harvest, whereas growing on straw reduces greatly the time to harvest but requires pasteurization to kill any microorganisms that come with the mycelium. Grey oyster mushrooms also grow extremely well on rich cellulose coffee grounds [7]. This interesting feature enables the sustainable cultivation of grey oyster mushroom based on the repurposing of coffee leftovers. Growing on coffee grounds has quite a big advantage since it has already been pasteurized by the coffee brewing process, meaning that the growth bed is ready for inoculation.

\section{Background Studies}

To devise their own solution, the team started by performing a series of studies on the marketing, sustainable development and ethical perspectives of the project.

\subsection{Marketing}

In order to develop a marketing plan, the team started by performing a market analysis. The coffee, mushroom consumption and sustainable foods markets were taken into consideration to define the need for such a product. Moreover, the strengths, weaknesses, opportunities and threats of the business were identified using the SWOT framework. On top of this, the team conducted a survey to gain a better understanding of the target audience and their needs as consumers. The survey results helped to select and justify marketing strategies. The full results of the survey can be found in the project detailed report [8].

The team analysed existing solutions and competition to find a niche. The two main competitors found are:

- Mushroom kits, which allow the consumer to grow fresh mushrooms at home. The kits are composed of a plastic bag with fully colonised mycelium. The most common combination is grey oyster mushrooms in a coffee ground bed. The price ranges from $9 €$ to $20 €$.

- Mushroom farms, which provide fresh, packed or canned mushrooms. Mushroom farms usually operate on a large scale in terms of quantity, variety and markets. 
The team decided to differentiate its product from other growth kits not only by reusing coffee waste and other materials, but also by creating a service surrounding the growth kit, offering bucket refilling with fully colonised mycelium and access to learning resources, such as workshops, monitoring systems and community forum.

Next, the team performed customer segmentation, considering geographic, demographic, behavioural and psychographic aspects, followed by a marketing mix analysis, consisting of the four P (place, product, promotion and placement). The product will be dedicated to mushroom eaters concerned with the environment and interested in healthy organic food. The main headquarters of the farm and selling store would be on ISEP, where the cultivation of mushrooms, educational workshops and bucket selling will take place. The team decided to create three selling options to differentiate the offer: the customer can buy the bucket, the bucket with chamber or the bucket, chamber and sensors. The plan is also to create a loyal customer base where it would be possible to get discounts and return equipment with reduction. Promotion, which was considered to be a crucial aspect at the beginning of the business, should be conducted simultaneously on several platforms ranging from the website and social media to local cafeterias (to spread information, collect coffee grounds and create discounts for customers) and community (to disseminate the concept within schools, environmental organizations and restaurants).

\subsection{Sustainable Development}

Sustainability, which is about preserving life on Earth, requires individuals and businesses to embrace radical changes. One area where difference can be made is in product development. During the design phase, environmental impacts must be taken into consideration and the whole life cycle of a product needs to be analysed, adopting a circular approach. Businesses usually consider the environmental impact at the end of the product life cycle, which prolongs the problem instead of producing environmentally friendly solutions. Environmentally conscious businesses are implementing other sustainable concepts and practices that have a more significant impact on the environment, emphasizing the principles of maximum value with minimum resources.

The mushroom farm project incorporates circular economy practices. The idealised company provides a subscription-based growth kit selling service. The kits are reused plastic buckets, originally from food industry, filled with inoculated coffee grounds. After harvest, the plastic bucket is to be returned to the company for reuse. The collection of coffee grounds and the product distribution are conducted locally to minimize the negative impacts of long transportation lines, minimising pollution. Using coffee leftovers and reusing buckets keeps waste to a minimum. The manual preparation of the growth kits contributes to the low energy consumption of the process.

\subsection{Ethical and Deontological Concerns}

The goal was to design an environmentally friendly product, adopt ethical advertising practices, and minimise liability risks (by designing a product compliant with public safety, health and welfare regulations).

For the idealised company to have a reliable and trustworthy reputation, treating customers fairly and advertising the service and product in the most transparent and honest way possible is of high importance. The environmental impact of the proposed solution is low due to component reuse and the adopted circular economy approach. In terms of liability risks and to prevent causing damages or accidents to the user, the team decided to comply with the applicable EU directives and existing food directives in Portugal.

\section{Proposed Solution}

Based on the previous studies, the team started by creating a concept map (Figure 1). The decision was to create an almost zero waste mushroom farming system supported by a circular sustainable process which reuses leftovers of food industry. The proposed solution consists, then, in growing grey oyster mushrooms in coffee grounds inside repurposed or reused plastic food containers. This solution, which is scalable, can be deployed both at the domestic and industrial levels. By growing mushrooms at home, individuals have access to fresh, healthy and organic food, contributing to improve the quality of life. The growth kits also allow small and medium size scale cultivation at home.

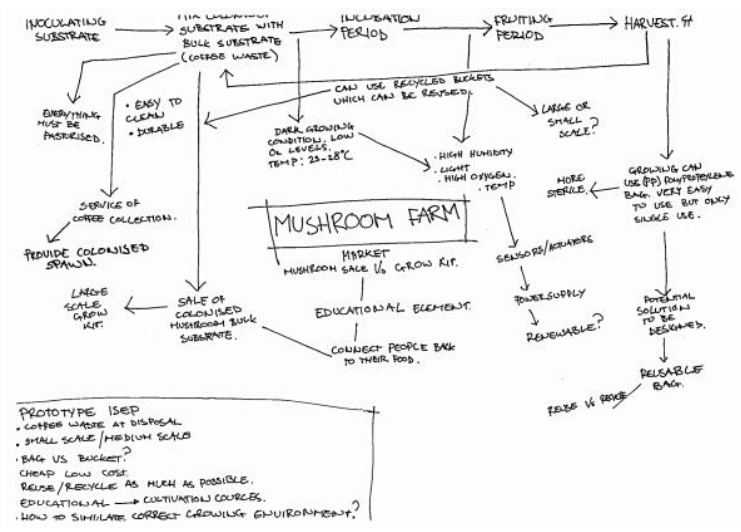

Figure 1 - Concept map

\subsection{Design}

Based on the described idea, the team decided to create Waste to Fungi, a circular economy business and product. It was designed around two mottos: reuse and repurposing (Figure 2). 


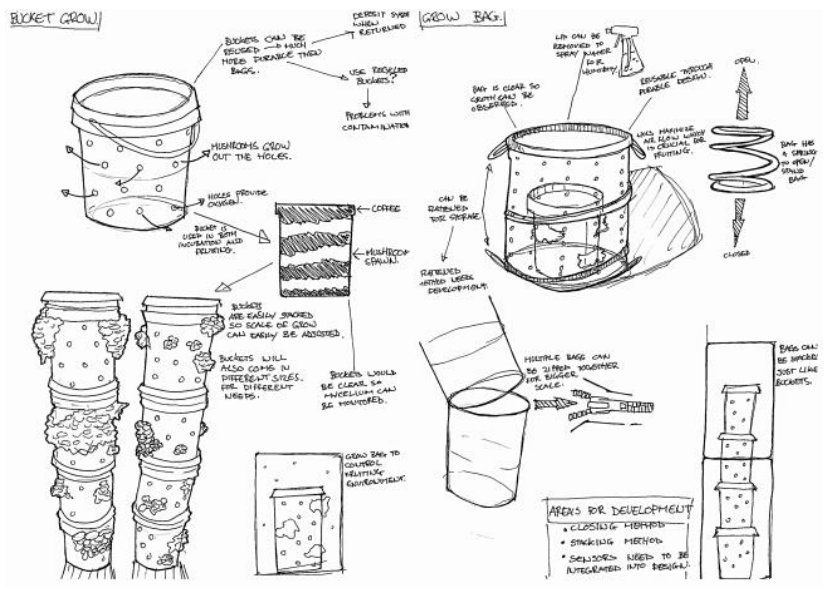

Figure 2 - Bucket and chamber concepts

In terms of reuse, the team goal was to reduce waste to a minimum. Opposed to standard mushroom producers who use plastic bags for the cultivation process once, the team decided not only to reuse food industry plastic buckets, but also to provide a recolonization service, where the buckets can be repeatedly refilled and sold with colonised mycelia.

The second aim was to create a more circular economy by filling the existing gap between waste and trash. This was achieved by repurposing and giving value to the waste of others.

The basic product component is the plastic bucket containing oyster mushroom colonised mycelium. Made of repurposed buckets, it can be reused or recycled, when it becomes too damaged. Alongside, the team developed a chamber which enables the user to achieve optimal growth conditions by controlling and adjusting temperature and humidity. The educational service offers information on the cultivation process, recipes and benefits of growing and eating mushrooms. This is not only an enriching and rewarding process, but expands people awareness and knowledge of sustainable food systems. This may encourage more environmentally friendly practices.

\subsection{Cultivation}

Grey oyster is the best suited mushroom variety to grow on coffee grounds since its cultivation requires cellulose, which abounds on coffee grounds. The team procured Pleurotus Ostreatus grain spawn from a local supplier.

Recycled food buckets were collected from cafeterias and restaurants around the production point. The buckets were cleaned and the sides drilled to create a uniform pattern of $10 \mathrm{~mm}$ holes for the mushrooms to grow. The coffee grounds were collected using plastic zip-lock bags.

The coffee grounds were mixed with hay with a ratio of 1:5. For guaranteed results the team decided to fully sterilize the substrate in an autoclave. The substrate mix was placed into $2 \mathrm{~L}$ glass beakers into the autoclave and heated to $121{ }^{\circ} \mathrm{C}$ for a total of $20 \mathrm{~min}$. Once the substrate cooled back to room temperature, $3 \mathrm{~kg}$ of substrate mix was mixed with $100 \mathrm{~g}$ of grain spawn. Before buckets were filled with the mix, they were cleaned with $70 \%$ Ethanol and holes were sealed with breathable tape.

In order to have comparative results the team decided to use a control group where the coffee in the substrate mix was not sterilized in the autoclave. This not only provided a control group, but also helped to determine whether full sterilisation is necessary. The prepared buckets were placed into a dark environment at a temperature of around $20{ }^{\circ} \mathrm{C}$, where they remained until the substrate was fully colonised by the mycelia. The team monitored daily the growth and contamination levels. Once the substrate mix was fully colonised by the mycelium, the buckets were placed in the fruiting chamber. The chamber maintains a high humidity environment (60\% to $80 \%$ ) around a temperature of $24{ }^{\circ} \mathrm{C}$ (within a range from $20^{\circ} \mathrm{C}$ to $28^{\circ} \mathrm{C}$ ). Humidity and temperature are monitored and adjusted with the help of the developed monitoring system. Within a week or two, the first set of mushrooms are matured.

The mushrooms can be handpicked with a twisting motion. All mushrooms must be removed for the process to restart. Typically, each colonised bucket provides three to four harvests. Once the fruiting ends, the bucket is removed from the fruiting chamber and is ready for recolonization. The substrate is removed and mixed into new substrate so the mycelium within the substrate can be recycled. Buckets are cleaned and stored for future use.

\section{Waste to Fungi Development}

Figure 3 displays the different actors involved in the devised circular economy proposal:

- $\quad$ Restaurant and cafeterias (Coffee Grounds) provide coffee brewing leftovers.

- Waste to Fungi (Logistics) provides transportation by electric cargo bikes, allowing the collection of coffee waste, buckets and other materials in the most sustainable and effective way.

- Waste to Fungi (Coffee Inoculation) mixes the mycelium and coffee grounds and tracks contamination for the safety of the customer.

- Customers (Growing and Harvesting) grow and harvest, using the buckets and chamber.

- Customers (Bucket Return) return used buckets for reuse.

- Waste to Fungi (Waste Reuse) provides the organic waste as fertilizer to cultivate coffee or other cultures.

- Waste to Fungi (Complementary Services) promotes sustainable food awareness and offers information about mushroom cultivation, benefits and recipes. 


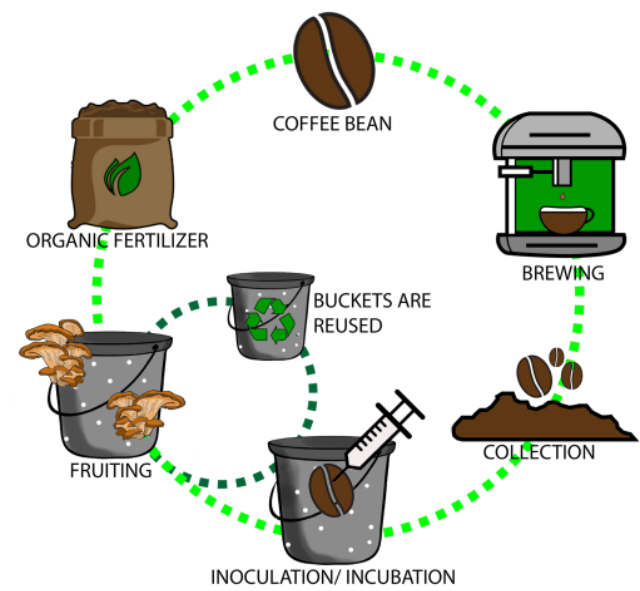

Figure 3 - Circular economy diagram

The project requirements included the automatic monitoring and control of the chamber conditions for medium or small scale deployments, low-cost hardware solutions, open source software, compliance with the International System of Units and applicable EU Directives ([9], [10], [11], [12] and [13]) and a maximum budget of $100 €$ for the construction of prototype.

The buckets, in Figure 4, are made of plastic packaging that has already been used at least once in the food industry. They have holes to enable fresh air supply for the mycelium development and for the mushroom to fruit.

The chamber, in Figure 4, allows to simulate a suitable environment for the fruiting and harvesting phases of the mushroom process. It regulates the humidity and temperature, creating an airflow and allowing indirect light to help the mushroom growth. This is made using fans, heaters and an automatic sprinkling system.
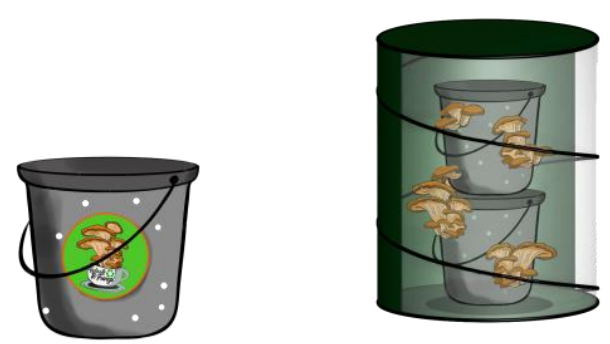

Figure 4- Bucket and chamber

The box diagram of the system is shown in Figure 6. The control system activates the actuators according to the chamber temperature and humidity values. Two fans are used to increase the air flow. The use of the sprinkler together with the fans cools the chamber, whereas the use of the power resistors with the fans warms the chamber. The sprinkler is actuated trough a servo motor. The system maintains the temperature and relative humidity of the chamber within the desired ranges, facilitating the growth of the mushrooms. Finally, with the help of a Wi-Fi module, the sensor data are sent to the cloud, making it possible to carry out studies on the ideal conditions for cultivating mushrooms.

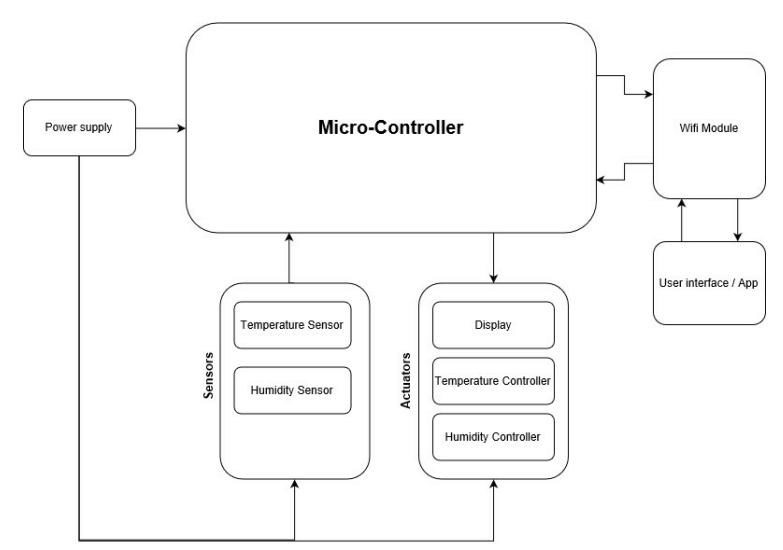

Figure 6 - Control system box diagram

\section{Tests and Results}

In order to ensure the proper functioning of the final prototype, several tests were performed.

\subsection{Electronics}

The monitoring system requires hardware and software testing. The different components of the electrical circuit were first checked separately and, then, together. The code was tested function by function and, in case of error, it was debugged and refined until the desired results were achieved.

The software tests included the data acquisition and data storage in the cloud. The assembled control system was tested in a room and inside the prototype chamber. In the first test, shown in Figure 7, the prototype was placed in a room with low light, temperature between $18{ }^{\circ} \mathrm{C}$ and $23^{\circ} \mathrm{C}$, and a water bucket to keep the humidity between $40 \%$ and $60 \%$.

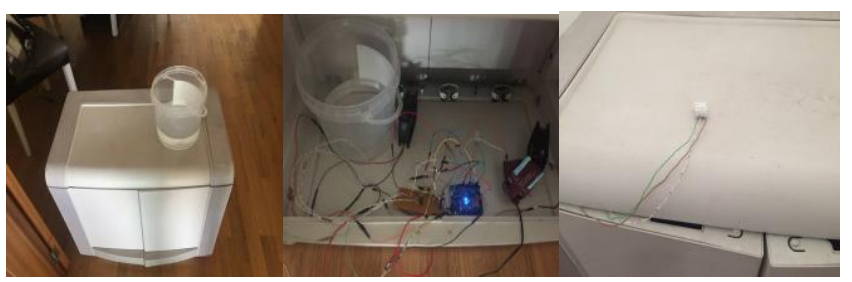

Figure 7 - Room setup

The objective was to verify if the system could properly monitor the temperature and humidity of the place. The tests started at 2019-06-08 16:17:20 and finished at 2019-06-09 10:34:22. The team managed to keep track of the parameters for about $17 \mathrm{~h}$, as can be seen in Figure 8 . 


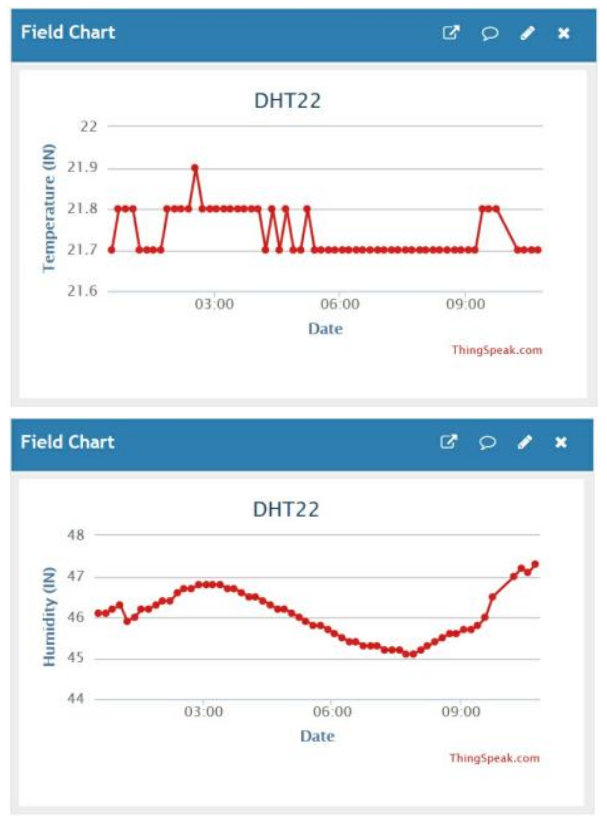

Figure 8 - Results for the room setup

The results indicated the proper operation of the acquisition system.

In the second case, the control system was placed in the chamber as shown in Figure 11. The tests started at 2019-06-16 18:45:17 and finished at 2019-06-16 19:10:21. The team managed to actuate on the system for about $1 \mathrm{~h}$. Figure 12 displays the results, showing that the temperature and relative humidity ranges. The relative humidity was slightly above the desired range because the average relative humidity on that day was $76 \%$.

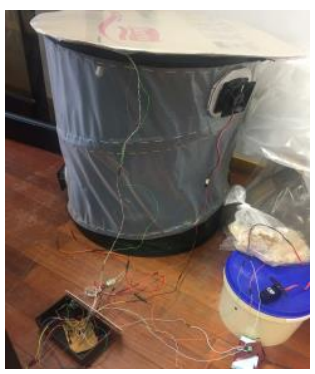

Figure 11- Chamber setup

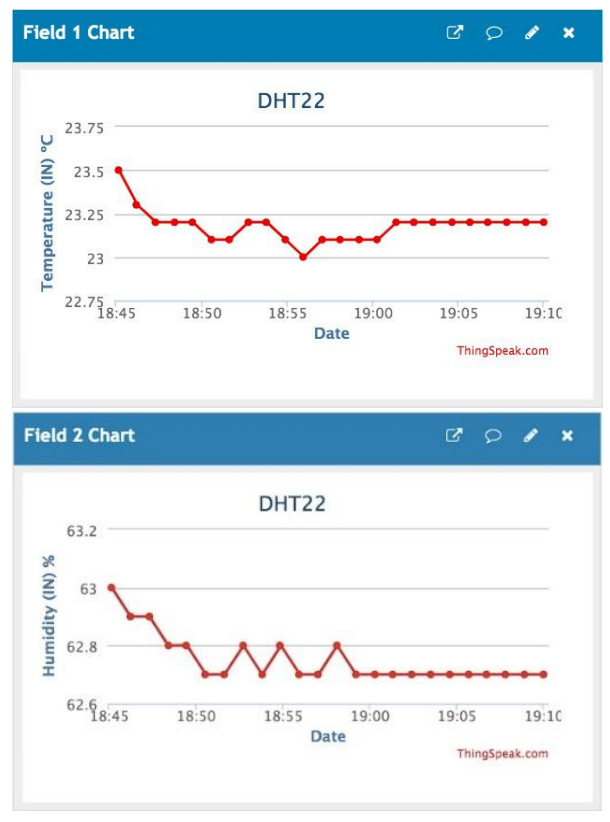

Figure 12 - Results for the chamber setup

\subsection{Cultivation}

The cultivation experiments were monitored and photographed daily. The goal was to follow closely the growth kits, looking for signs of contamination. Whenever contamination occurred, the infected containers were isolated, kept under observation and the causes investigated. Since mushroom cultivation is a long process, it is important to maintain the right temperature and relative humidity conditions in the cultivation room. The complete set of results can be found in the project report [8].

The first cultivation batch was produced on 2019-05-07. Figure 13 shows the recorded temperature and humidity during a fourweek period. Figure 14 displays a series of photos showing the mycelium development from the first to the fourth week. The results of the first batch of buckets were inconclusive. One of the non-pasteurized control buckets showed early signs of green mould, most likely caused by pre-contaminated stale coffee grounds. The buckets with non-pasteurized substrate were more successful. The buckets with pasteurized substrate were contaminated with neurospora due to the high level of internal humidity of the pasteurized substrate (introduced during the autoclave sterilisation), the increase in humidity and temperature in the second week and poor ventilation of the initial incubation site. 


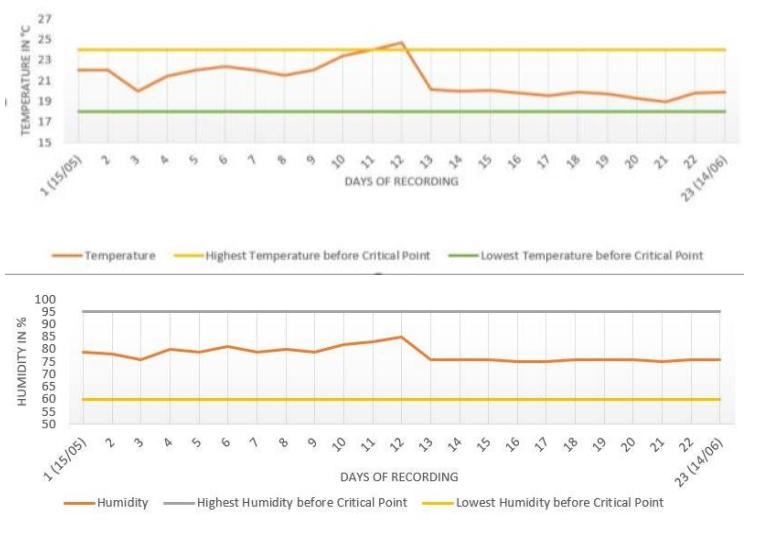

Figure 13 - Temperature and humidity at the incubation site
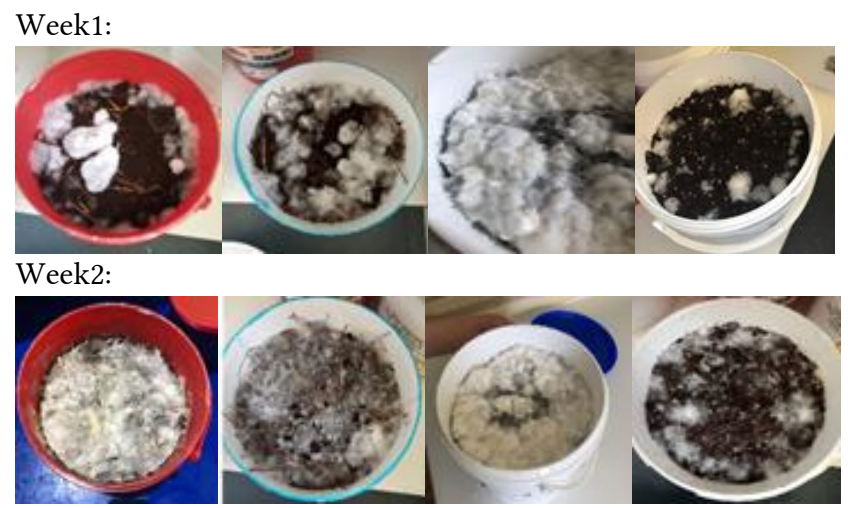

Week3:

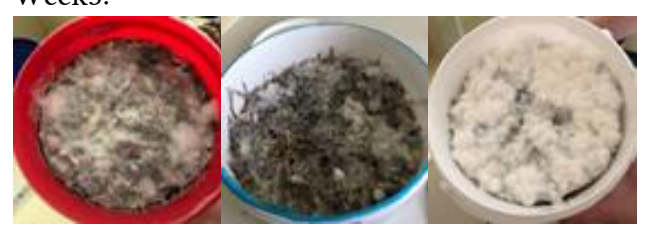

Week4:

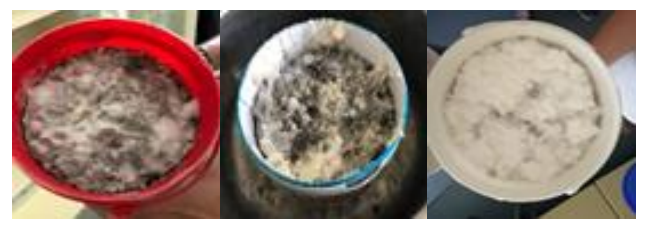

Figure 14 - Cultivation process

For the second batch, the team changed the incubation site to a new area where temperature and humidity remains within an appropriate range and with better ventilation. The results were very promising, with the first bucket beginning to fruit (Figure $15)$.
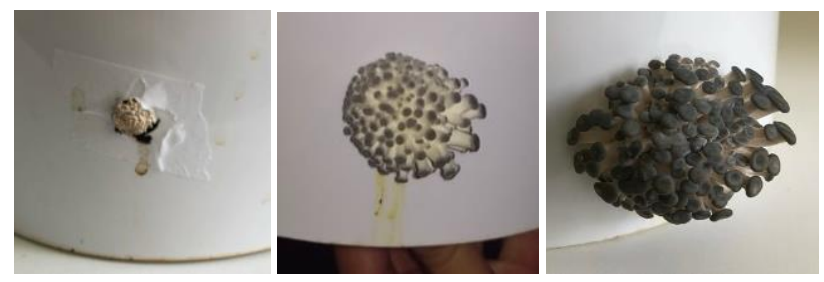

Figure 15- First 3 days of fruiting

\section{Conclusion}

This project explored the feasibility of creating a successful business based on the idea of using coffee leftovers to grow oyster mushrooms. It involved the creation of a marketing plan to promote the growth kit and the surrounding services to target consumers; a detailed business plan to address logistics, costs and funding, supplies and target consumers; the learning and understanding of the cultivation process; and the design, assembly and test of a controlled fruiting chamber. The cultivation process, despite initial contamination problems, provided the team with a sound understanding of the process and the potential risks associated.

The team would like to investigate further the potential sources of contamination to develop preventive countermeasures with the help of an expert. Alternatively, the inoculation process could be outsourced, whereby the team would collect the raw materials for the growing substrate, while an external company (with more expertise in the field) would inoculate and colonize the buckets.

Collaborative work can be difficult, especially in a multicultural and multidisciplinary context. One of the biggest challenges the team faced was time management while organizing logistics and distributing tasks amongst the team members. The team worked with each member's strengths, consolidating their individual knowledge while developing other skills in subjects such as marketing, sustainability or ethics. Soft skills such as organization and communication were also practiced and developed. All the team members agreed that EPS@ISEP was a very useful experience not just for personal development, but also for future employment. Not to mention that the whole experience allowed the members to explore Portugal and indulge in its rich culture.

\section{ACKNOWLEDGMENTS}

The team would like to thank ISEP for the opportunity to take part in this great learning experience; the Laboratory of Autonomous Systems for the sponsoring and the microbiology technicians for the help and support provided.

This work was partially supported by the European Union (FEDER funds) through COMPETE and by National Funds through the Portuguese funding through the Portuguese funding agency, Fundação para a Ciência e a Tecnologia (FCT), under Project UID/QUI/50006/2019 and Project UID/EEA/50014/2019. 


\section{REFERENCES}

[1] B. Malheiro, M. Silva, M. C. Ribeiro, P. Guedes and P. Ferreira, 2015. The European Project Semester at ISEP: The Challenge of Educating Global Engineers. European Journal of Engineering Education, 40(3), 328-346. DOI: http://dx.doi.org/10.1080/03043797.2014.960509.

[2] A. J. Duarte, B. Malheiro, E. Arno, I. Perat, M. F. Silva, P. Fuentes, P. Ferreira \& P. Guedes, 2019. Engineering Education for Sustainable Development: The European Project Semester Approach. IEEE Transactions on Education. DOI http://dx.doi.org/10.1109/te.2019.2926944.

[3] Markets, Markets, Mushroom Market by Type (Button, Shiitake, and Oyster), by Application (Fresh Mushrooms and Processed Mushrooms (Dried, Frozen, and Canned)), \& by Region - Global Trends \& Forecast to 2019 https://www.marketsandmarkets.com/Market-Reports/mushroom-market733.html.

[4] A. Sayner, 2019, GroCycle, Growing Mushrooms in Coffee Grounds https://grocycle.com/growing-mushrooms-in-coffee-grounds/.

[5] Statista, Coffee - Portugal | Statista Market Forecast, https://www.statista.com/outlook/30010000/147/coffee/portugal.

[6] M. Ammaiyappan, 2015, How to cultivate mushrooms, https://amgurumushrooms.blogspot.com/2015/10/
[7] A. Sayner, 2019, GroCycle, setting up a low tech mushroom farm, https://grocycle.com/how-to-set-up-a-low-tech-mushroom-farm/

[8] Alexander Winter, Erendiro Pedro, Julia Ślasko, Julien Battaglini, Mäike Faelker. and Ronald Kivipelto, 2019. Mushroom Farming Report, http://www.eps2019-wiki3.dee.isep.ipp.pt/doku.php?id=report.

[9] European Commission. Machine Directive (2006/42/CE 2006-05-17) http://ec.europa.eu/growth/sectors/mechanicalengineering/machinery/index en.htm.

[10] European Commission. Electromagnetic Compatibility Directive (2004/108/EC 200412 15), http://ec.europa.eu/growth/sectors/electrical-engineering/emcdirective/index en.htm.

[11] European Commission. Low Voltage Directive (2014/35/EU 2016-04-20), http://ec.europa.eu/growth/sectors/electrical-engineering/lvddirective/index_en.htm.

[12] European Commission. Radio Equipment Directive (2014/53/EU 2014-04-16), http://ec.europa.eu/growth/sectors/electrical-engineering/rttedirective/index en.htm.

[13] European Commission. Restriction of Hazardous Substances (ROHS) in Electrical and Electronic Equipment Directive (2002/95/EC 2003-01-27), http://ec.europa.eu/environment/waste/rohs_eee/legis_en.htm. 